(C) 2017 IEEE. Personal use of this material is permitted. Permission from IEEE must be obtained for all other uses, in any current or future media, including reprinting/republishing this material for advertising or promotional purposes, creating new collective works, for resale or redistribution to servers or lists, or reuse of any copyrighted component of this work in other works. 


\title{
Fuzzy-Based Self-Tuning Model Predictive Direct Power Control of Grid-Connected Multilevel Converters
}

\author{
Mahlagha M. Aghdam, Student Member, IEEE, Ricardo P. Aguilera, Member, IEEE, Li Li, Member, IEEE, \\ Jianguo Zhu, Senior Member, IEEE \\ Centre of Green Energy and Vehicle Innovation, University of Technology Sydney, Ultimo, NSW 2007, Australia \\ Mahlagha.MahdaviAghdam@student.uts.edu.au
}

\begin{abstract}
This paper proposes a self-tuning model predictive direct power control (MPDPC) strategy for power flow control and power quality improvement in gridconnected power converters. At each sampling instant, a fuzzy logic controller is used to determine online the best weighting factor values for a given operating point. These values are then used to solve the multi-objective optimal control problem associated to the MPDPC. The optimal solution that minimizes the multi-objective cost function is chosen as the input (power switch state). The proposed method is examined through a case study and verified numerically via MATLAB SIMULINK. A comparative study is conducted to demonstrate the effective performance of this approach. As a result of the proposed weighting factor online tuning, an improved performance in terms of total harmonic distortion and average switching frequency is attained when compared with fixed weighting factors.
\end{abstract}

Index Term--Fuzzy model predictive control; Gridconnected power converters; Power flow control; Weighting factor.

\section{INTRODUCTION}

In the power electronics field, research is focused not only on obtaining novel power converter topologies, but also on deriving advanced control strategies for power converters. The advancement of modern processing and devices opened a new horizon to power converter control. In this area, model predictive control (MPC) has emerged as a promising control alternative when compared to the traditional PWM-based linear controllers [1,2]. MPC is a well-established method and its concepts, operating principles are studied in [3]. Furthermore, its technology readiness for real-time implementation in distributed generation applications is assessed in [4].

Among power converters, multilevel converters (MLCs) achieved vast industrial success for medium voltage and high power applications, such as variable speed drive, distributed generations, and active power filter [5]. The neutral point diode clamped (NPC) [6, 7], flying capacitor [8, 9], and cascaded H-bridge [10] power converters emerged as prominent topologies for the mentioned applications. MLCs offer improved harmonic performance and higher power capabilities compared with the two-level converters [11].

Due to the limited number of switching states in power converters, finite control set MPC (FCS-MPC) is a suitable approach to predict the future system behavior. One of the most suitable MPC strategies for distributed generation systems is the so-called model predictive direct power control (MPDPC). To control the power flow, MPDPC employs the active and reactive powers as control variables.

While FCS-MPC carries some benefits, the algorithm needs to be reformed for various purposes, mostly due to the variety of the plant characteristics that causes some challenges for the design. A multi-objective optimization problem can be formulated to take into account different requirements and constraints with corresponding weighting factors. Therefore, all the control necessities will be observed by the controller simultaneously.

Nevertheless, the weighting factor adjustment is generally addressed by heuristic approaches for which there is not a detailed analytical study [12]. To overcome this challenge, the selection of weighting factors is avoided, in some cases via a nontrivial process based on a ranking approach in [13, 14]. By using this method, multiple voltage vectors may have the same average ranking. Although priorities can be allocated for each objective to overcome this matter, it remains an open discussion in this approach. Furthermore, it also increases the computational burden up to three times more than the heuristic methods. In [15] the weighting factors are eliminated by converting the objectives to the same nature. Moreover, fuzzy decision making approach is utilized and developed in [16, 17], where a membership function represents the degree of achievement of the target.

In this work, an FCS-MPDPC combined with a fuzzy logic control (FLC) approach with several objectives for grid-connected power converters is proposed. This method uses an FLC scheme to regulate the fitting value of weighting factors online subject to the specific dynamic conditions, leading to improved power system performance. Based on the simulation results, it is shown that with the variable weighting factors, corresponding to each operating condition, better transient and steady state performance can be achieved.

The rest of this paper is organized as follow. The case study based on FCS-MPDPC is described in Section II. The proposed approach and its steps are discussed in Section III. Section IV is devoted to the simulation results, discussion, and comparison with heuristic and ranked-based approaches. Section $\mathrm{V}$ is dedicated to the conclusion and future work. 


\section{CASE Study}

A grid-connected three-level three-phase neutral point clamped (3L-3Ph NPC) inverter is one of the most common inverters for medium power applications. The topology of the $3 \mathrm{~L}-3 \mathrm{Ph}$ NPC grid-connected inverter is presented in Fig. 1. The inverter consists of six complimentary pairs of IGBT switches. As we know, for $r$-level $s$-phase $(r \mathrm{~L}$-s $\mathrm{Ph})$ power converters, there are $m=$ $r^{s}$ possible voltage vectors. Therefore, there are 27 possibilities, including three zero, twelve small, six medium and six large voltage vectors as illustrated in Fig. 2. The output voltage of the inverter in the stationary frame is described by

$$
\begin{gathered}
V_{m}^{\alpha \beta}=\frac{V_{D C}}{2} M S_{m} \\
S_{m}=\left[\begin{array}{lll}
S_{a m} & S_{b m} & S_{c m}
\end{array}\right]^{T}
\end{gathered}
$$

where $\mathrm{M}$ is the Clarke transformation matrix, provided in the Appendix, $V_{D C}$ is the DC-link voltage and $S_{m}$ is the switching signal for $m=0, \ldots, 26$. The mathematical model of $3 \mathrm{~L}-3 \mathrm{Ph}$ NPC grid-connected inverter can be formulated in the stationary frame via matrix $M$ as

$$
V_{m}{ }^{\alpha \beta}=L \frac{d i_{o}^{\alpha \beta}}{d t}+R \cdot i_{o}^{\alpha \beta}+V_{g r i d}^{\alpha \beta}
$$

where $i_{o}$ is the output current of the inverter and $V_{\text {grid }}$ is the main grid voltage. Furthermore, for a balanced and positive sequence system, the active power $P$ and reactive power $Q$ can be obtained as

$$
\begin{aligned}
& P=\frac{3}{2}\left({V_{\text {grid }}}^{\alpha} i_{0}^{\alpha}+V_{\text {grid }}{ }^{\beta} i_{0}^{\beta}\right) \\
& Q=\frac{3}{2}\left(V_{\text {grid }}{ }^{\beta} i_{0}^{\alpha}-V_{\text {grid }}^{\alpha} i_{0}^{\beta}\right)
\end{aligned}
$$

By taking the first derivative from (3), the system model can be obtained based on the active and reactive power. The system model is in the non-linear form; therefore, to predict the future behavior of the system, the linearization approach is applied. The state-space model of the system can be obtained as,

$$
\frac{d x}{d t}=A \boldsymbol{x}+B \boldsymbol{u}
$$

The matrices $A$ and $B$ are provided in the Appendix, where the state variables, input variable are,

$$
\begin{gathered}
\boldsymbol{x}=\left[\begin{array}{llll}
P & Q & V_{\text {grid }}{ }^{\alpha} & V_{\text {grid }}{ }^{\beta}
\end{array}\right]^{T} \\
\boldsymbol{u}=\left[\begin{array}{llll}
V_{m}{ }^{\alpha} & V_{m}{ }^{\beta}
\end{array}\right]^{T}=M \frac{V_{D C}}{2}\left[\begin{array}{lll}
S_{a m} & S_{b m} & S_{c m}
\end{array}\right]^{T}
\end{gathered}
$$

In this topology, balance of the neutral point is a compulsory action for achieving the high power quality. There are two capacitors in this topology which serve as a voltage divider of full DC-link $\left(V_{D C}=V c_{1}+V c_{2}\right)$.

$$
\begin{aligned}
& C_{1} \frac{d V c_{1}}{d t}=I_{D C}-i_{1}-\frac{V c_{1}}{R_{C 1}} \\
& C_{2} \frac{d V c_{2}}{d t}=I_{D C}-i_{2}-\frac{V c_{2}}{R_{C 2}}
\end{aligned}
$$

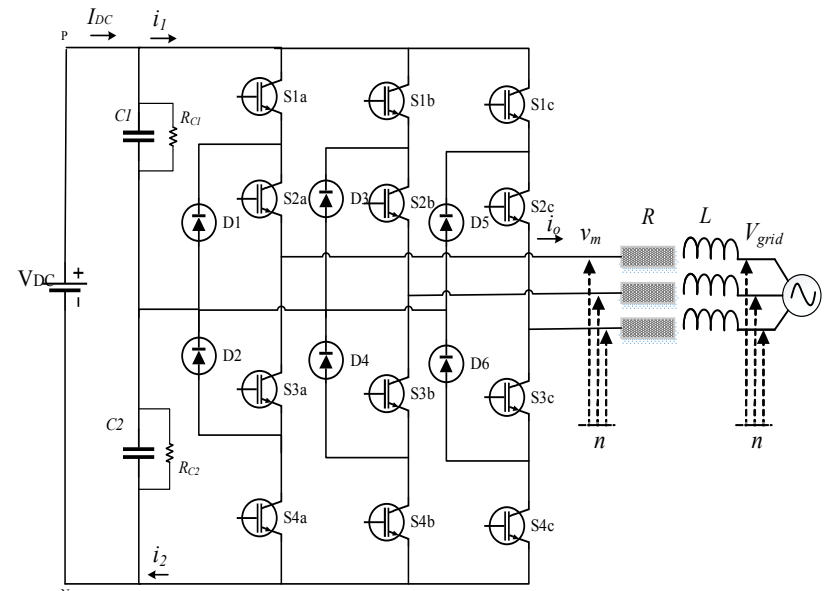

(a)

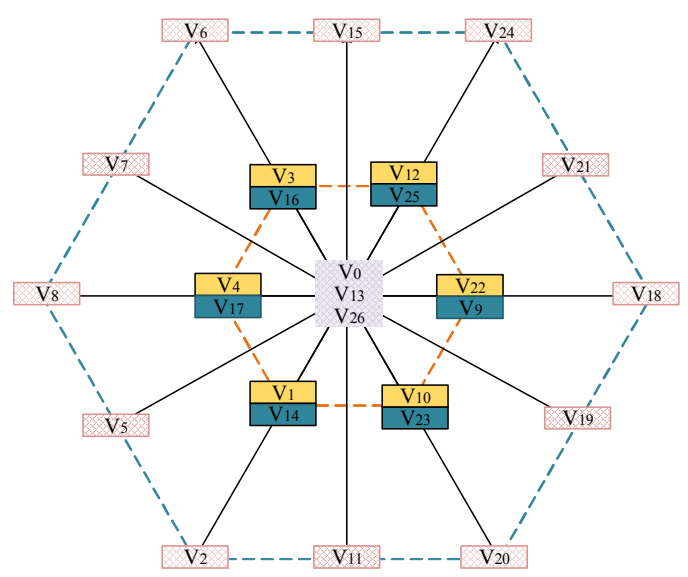

(b)

Fig. 1. 3L-3Ph NPC grid-connected inverter a) Topology b) Voltage vectors

where $i_{1}$ and $i_{2}$ are the inverter input currents computed based on the switching signals and phase currents at each sampling time.

The state-space model of the DC-link capacitor voltages can be obtained below and the matrices $D, F$ and $G$ are provided in the Appendix.

$$
\begin{gathered}
\frac{d y}{d t}=D \boldsymbol{y}+F \boldsymbol{w}+G I_{D C} \\
\boldsymbol{y}=\left[\begin{array}{llll}
V_{C 1} & V_{C 2}
\end{array}\right]^{T} \\
\boldsymbol{w}=\left[\begin{array}{lll}
i_{1} & i_{2}
\end{array}\right]^{T}=\left[\begin{array}{lll}
i_{a} & i_{b} & i_{c}
\end{array}\right]\left[\begin{array}{lll}
S_{a m} & S_{b m} & S_{c m}
\end{array}\right]^{T}
\end{gathered}
$$

As a result, for the power flow control and switching frequency reduction, the cost function will address three control objectives: active and reactive power tracking, capacitor voltage balancing, and switching loss minimization.

The block diagram of the FCS-MPDPC for a $3 \mathrm{~L}-3 \mathrm{Ph}$ NPC inverter is depicted in Fig. 2. The mathematical model of this case can be formulated in the stationary frame. The state variable is $\boldsymbol{z}=\left[\begin{array}{ll}\boldsymbol{x} & \boldsymbol{y}\end{array}\right]^{T}$ and the input variable is $S_{m}=\left[\begin{array}{lll}S_{a m} & S_{b m} & S_{c m}\end{array}\right]^{T}$. 


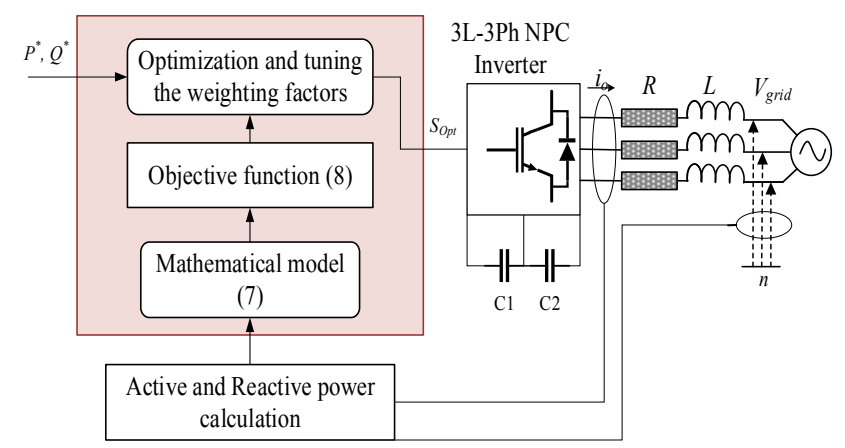

Fig. 2. Block diagram for FCS-MPDPC of 3L-3Ph NPC grid-connected inverter

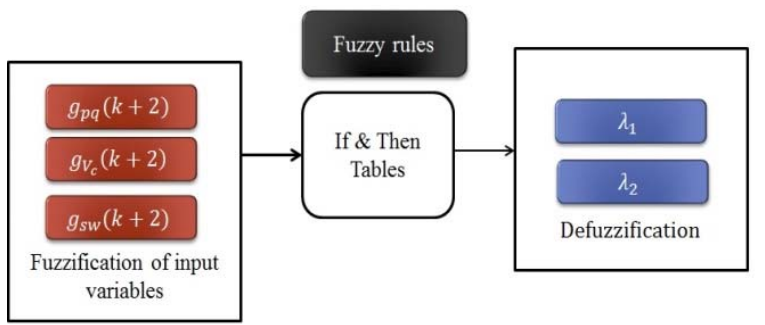

Fig. 3. Fuzzy logic control technique

After discretizing and shifting one step forward, the future value of balanced voltage will be obtained. The cost function has three terms with each addressing one objective as the following,

where,

$$
\begin{gathered}
J(k)=g_{P Q}(k+2)+\lambda_{1}(k) g_{V_{c}}(k+2) \\
+\lambda_{2}(k) g_{s w}(k+2)
\end{gathered}
$$

$$
\begin{aligned}
& g_{P Q}(k+2)=\left(P(k+2)-P^{*}\right)^{2}+\left(Q(k+2)-Q^{*}\right)^{2} \\
& g_{V_{c}}(k+2)=\left(V c_{1}(k+2)-V c_{2}(k+2)\right)^{2} \\
& g_{s w}(k+2)=\left(V_{m}(k+1)-V_{m}(k)\right)^{2}
\end{aligned}
$$

\section{FUZZY-BASED SELF-TUNING FCS-MPDPC}

The FLC is employed to adjust the weighting factor of the optimization problem for predicting the optimal switching pattern of the next sampling time. Every fuzzy inference system (FIS) is composed of three stages including fuzzification, fuzzy rules, and defuzzification as described in Fig. 3. The membership functions (MFs) are the essential part of FLC. It can be an arbitrary curve with the shape defined as a function that suits designers in approaching simplicity and efficiency. A degree of membership is given to the inputs through MFs.

The flowchart of the proposed fuzzy-based self-tuning FCS-MPDPC is illustrated in Fig. 4. To evaluate and minimize the multi-objectives function, for each operating point, specific weighting factors are required. In this case study, for each objective, i.e., active and reactive power tracking, capacitor voltage balancing, and switching loss minimization, a triangle MF is chosen due to its simplicity.

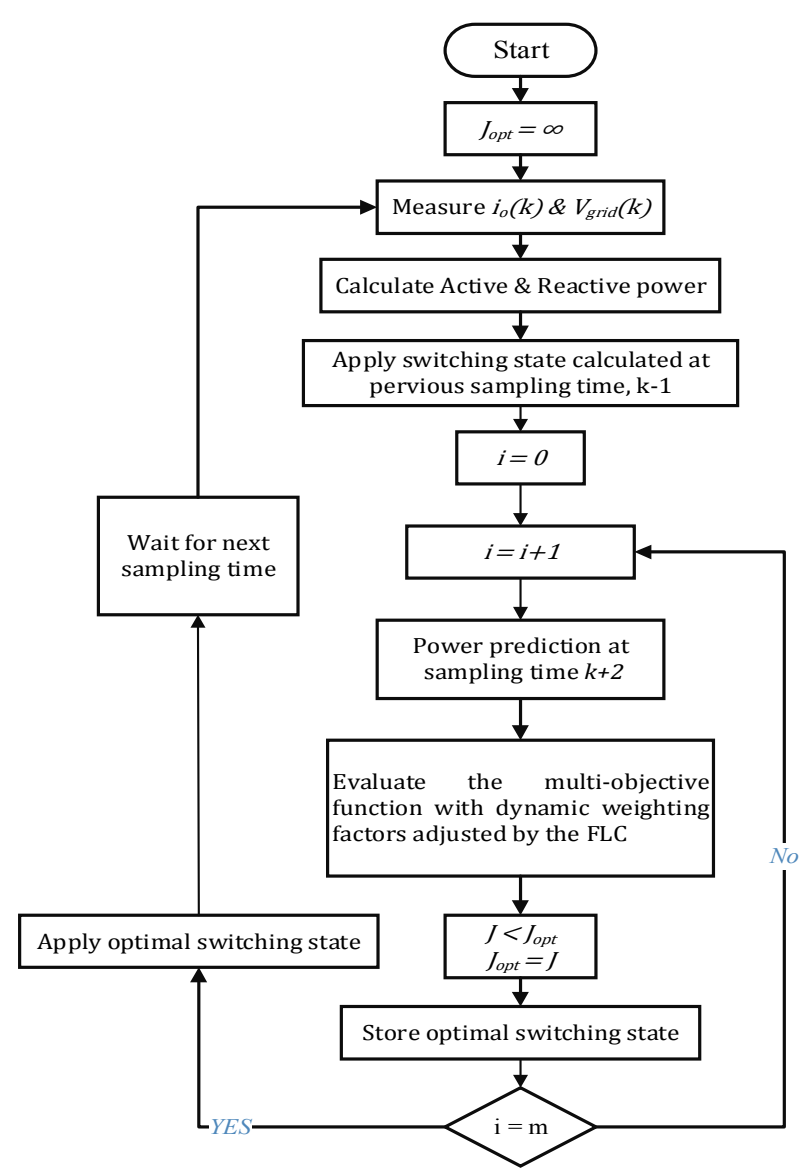

Fig. 4. Flowchart of proposed method

The domain of discourse for each of them is divided into five parts. Each MF maps the error to the range between 0 and 1. Rules are defined based on Table I for $\lambda_{1}$; Similar rule can be defined for $\lambda_{2}$ as well. The AND method for evaluating the rules and centroid technique are applied for defuzzification to get the numerical values of the weighting factors.

\section{RESUlTS, COMPARISON AND DISCUSSION}

\section{A. Simulation Results}

A 3L-3Ph NPC gird-connected inverter with RL filters and loads is simulated by using MATLAB/Simulink. To show the effectiveness of the proposed method, besides the FLC, the weighting factors are also designed through try and error [18] as well as fuzzy decision making (FDM) scheme $[16,17]$ for the comparison purpose. The system parameters are listed in Table II.

The results are presented for 3 cases. In case 1, the weighting factors are considered fixed, while in cases 2 and 3, the FDM and FLC adjust the weighting factors based on each operating point, respectively. The active and reactive power references are set to zero, initially and then the active power reference is increased to $5 \mathrm{~kW}$ at $0.01 \mathrm{~s}$. After that, the active power reference is changed to -2 and $0 \mathrm{~kW}$ at 0.02 and $0.03 \mathrm{~s}$, respectively. The reactive power reference is changed to 2 and $-2 \mathrm{kVar}$ at $0.04 \mathrm{~s}$, $0.05 \mathrm{~s}$, correspondingly. 
TABLE I: FUZZY RULES FOR $\lambda_{1}$

\begin{tabular}{|c|c|c|c|c|c|c|}
\hline \multicolumn{2}{|c|}{$\lambda_{1}$} & \multicolumn{5}{c|}{$g_{P Q}(\boldsymbol{k}+2)$} \\
\cline { 3 - 7 } \multicolumn{2}{|c|}{} & VS & S & M & B & VB \\
\hline \multirow{3}{*}{$\boldsymbol{g}_{V_{c}}$} & VS & M & S & VS & VS & VS \\
\cline { 2 - 7 }$(\boldsymbol{k}+2)$ & S & B & M & S & VS & VS \\
\cline { 2 - 7 } & VB & B & M & S & VS \\
\cline { 2 - 7 } & B & VB & VB & B & M & S \\
\cline { 2 - 7 } & VB & VB & VB & VB & B & M \\
\hline
\end{tabular}

\begin{tabular}{lcc}
\hline & TABLE II: SYSTEM PARAMETERS \\
\hline Filter resistance & $R$ & $0.51 \Omega$ \\
Filter inductance & $L$ & $10 \mathrm{mH}$ \\
Grid voltage & $V_{\text {grid }}$ & $120 \mathrm{~V}$ \\
Voltage frequency & $f$ & $50 \mathrm{~Hz}$ \\
Sampling period & $T_{S}$ & $100 \mu \mathrm{s}$ \\
Capacitor & $C$ & $22 \mu \mathrm{F}$ \\
Resistance & $R_{C}$ & $0.1 \Omega$ \\
DC source voltage & $V_{D C}$ & $700 \mathrm{~V}$ \\
\hline
\end{tabular}
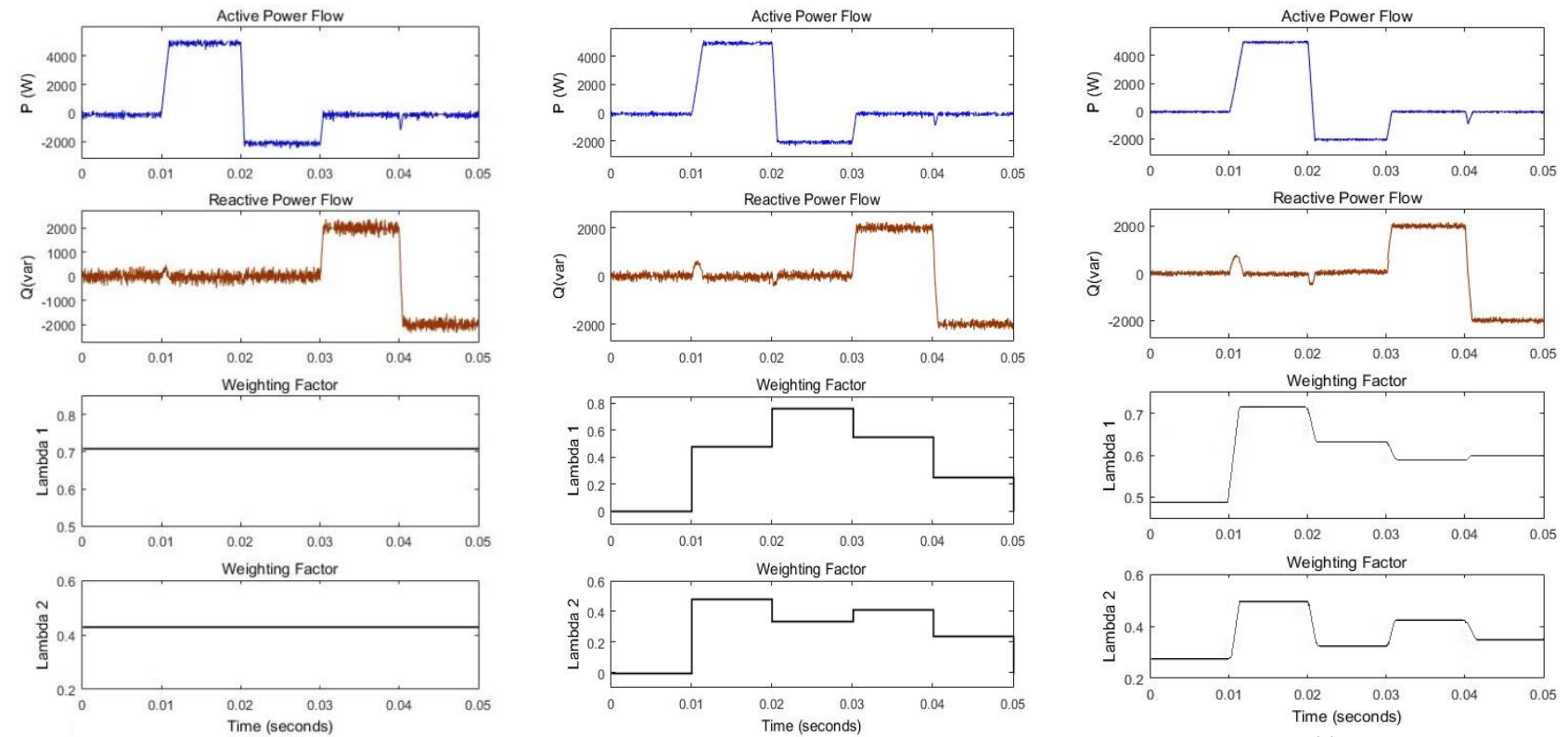

Weighting Factor

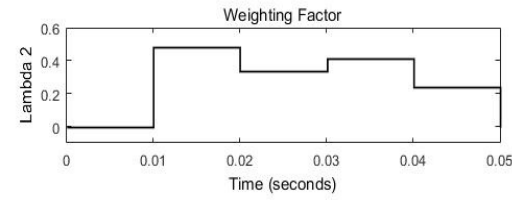

(b)

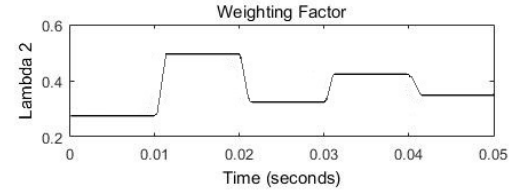

(c)

Fig. 5. Numerical simulation results for power flow control achieved by FCS-MPDPC for 3L-3Ph NPC grid-connected inverter a) Case 1: fixed value of weighting factors $\lambda_{1}=0.8, \lambda_{2}=0.1$ b) Case 2: fuzzy decision making method (c) Case 3: fuzzy logic control scheme

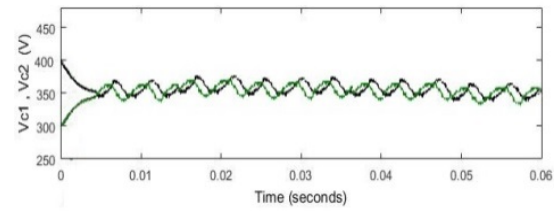

(a)

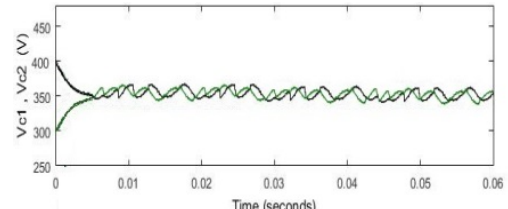

(b)

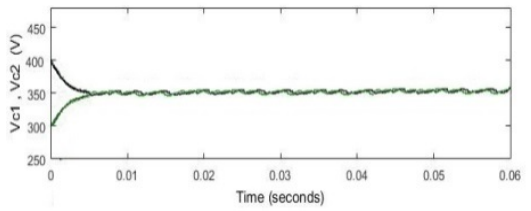

(c)

Fig. 6. Numerical simulation results for Capacitor voltage balancing achieved by FCS-MPDPC for 3L-3Ph NPC grid-connected inverter a) Case 1: fixed value of weighting factors $\lambda_{1}=0.8, \lambda_{2}=0.1$ b) Case 2: fuzzy decision making method (c) Case 3: fuzzy logic control scheme

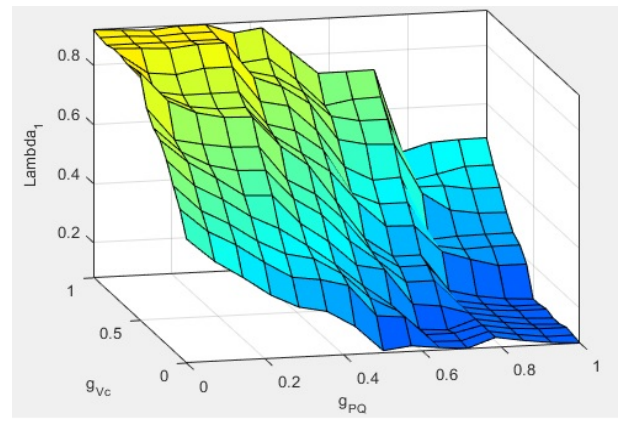

(a)

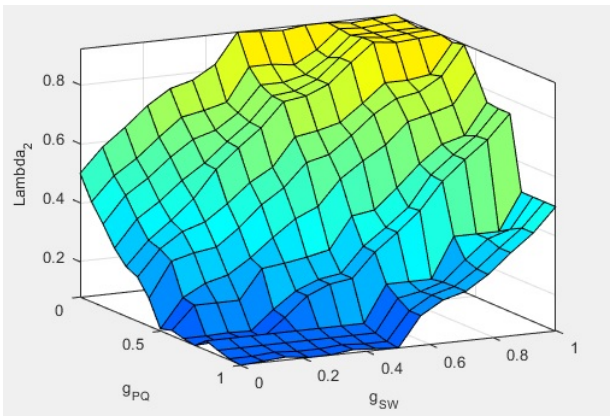

(b)

Fig. 7. Weighting factors surface curves (case 3) a) $\lambda_{1}$ b) $\lambda_{2}$ 
TABLE III: QUANTITATIVE COMPARISON

\begin{tabular}{|c|c|c|c|c|}
\hline \multirow{2}{*}{ Indices } & \multicolumn{4}{|c|}{ FCS-MPDPC } \\
\cline { 2 - 5 } & Heuristic & $\begin{array}{c}\text { Ranked- } \\
\text { based }\end{array}$ & $\begin{array}{c}\text { FDM- } \\
\text { Based }\end{array}$ & FLC \\
\hline $\boldsymbol{f}_{\boldsymbol{s}}(\mathrm{Hz})$ & $10 \mathrm{k}$ & $10 \mathrm{k}$ & $10 \mathrm{k}$ & $10 \mathrm{k}$ \\
\hline $\boldsymbol{f}_{\boldsymbol{s} \boldsymbol{w}}(\mathrm{kHz})$ & 2.102 & 1.854 & 1.5367 & 1.381 \\
\hline $\boldsymbol{P}_{\text {rip }}$ & 0.696 & 0.523 & 0.451 & 0.409 \\
\hline $\boldsymbol{Q}_{\text {rip }}$ & 0.791 & 0.512 & 0.423 & 0.347 \\
\hline THD (\%) & 8.457 & 5.847 & 4.066 & 3.67 \\
\hline $\begin{array}{c}\text { Settling } \\
\text { Time }\end{array}$ & 0.5982 & 0.4004 & 0.3752 & 0.3428 \\
\hline $\begin{array}{c}\text { Overshoot } \\
(\%)\end{array}$ & 47.146 & 40.11 & 38.98 & 37.54 \\
\hline $\begin{array}{c}\text { Calculation } \\
\text { Time }(\mu \mathrm{s})\end{array}$ & 58.7 & 69.3 & 76.8 & 89.1 \\
\hline W.F & Constant & Variable & Variable & Variable \\
\hline
\end{tabular}

Fig. 5 (a)-(c) show the power flow for a $3 \mathrm{~L}-3 \mathrm{Ph}$ NPC grid-connected inverter with FCS-MPDPC strategy. In Fig. 5 (a), case 1, the active and reactive power tracking is achieved through fixed weighting factors, $\lambda_{1}=0.8, \lambda_{2}=0.1$ for all the operating points. In case 2 , the FDM method which is characterized by use of the decision function examined as can be seen in Fig.5 (b). The power flow tracking and change of the weighting factors of the proposed method, case 3 , are provided in Fig.5 (C).

Fig. 6 (a), (b) and (c) show the neutral voltage balancing of 3L-3Ph NPC-VSI with FCS-MPDPC strategy for case 1, 2, and 3 correspondingly. Fig. 7 (a) and (b) show surface curves for the weighting factors attained by FLC, which is the graphical representation for the output of the FLC. The corresponding weighting factor for $g_{V_{c}}(k+2), \lambda_{1}$, is obtained based on the changes in $g_{P Q}(k+2)$ and $g_{V_{c}}(k+2)$. Similarly, $\lambda_{2}$ is adjusted based on the variations in $g_{P Q}(k+2)$ and $g_{\text {sw }}(k+2)$.

As can be seen, in the power flow tracking, the improvement in transient and steady state performance is shown in case 3 compared with case 1 . In order to get a better evaluation of the proposed control algorithm, the quantitative comparison is conducted next.

\section{B. Quantitative Comparison and Discussion}

A quantitative comparison of tuning weighting factors for the FCS-MPDPC strategy in this case study is carried out. The fuzzy-based self-tuning method is compared with other mentioned approaches. With the intention of leading to a better comparison of the approaches for tuning weighting factors in the multiobjectives MPC, the indices like average switching frequency, THD, and power ripples, overshoot, settling and calculation time are tabulated in Table III. The quantities are measured through MATLAB.

For clearer quantitative comparison, the indices are normalized. $P_{\text {rip }}$ and $Q_{\text {rip }}$ are the active and reactive power ripples which are obtained based on their standard deviation from the average one. The settling time is normalized by the value of $T_{s}$. It can be realized that by the FLC scheme, the power ripples are reduced by $21.19 \%$. Therefore, the unity power factor can be achieved, with a reduced power ripples. However, the calculation time for FLC-based method is increased, by approximately $43 \%, 38 \%$, and $24 \%$ in comparison with the heuristic, ranked-based, and FDM-based schemes, respectively.

Additionally, the proposed method shows a good performance in terms of the total harmonic distortion (THD) percentage and average switching frequency. In terms of the transient performance, the proposed strategy can achieve the fast dynamic response through the conventional MPDPC.

\section{APPENDIX}

$\mathrm{M}$, the Clarke transformation matrix, is defined below.

$$
M=\frac{2}{3}\left[\begin{array}{rrr}
1 & -\frac{1}{2} & -\frac{1}{2} \\
0 & \frac{\sqrt{3}}{2} & -\frac{\sqrt{3}}{2}
\end{array}\right]
$$

A, B, D, F and G are the space state matrices in the continuous-time domain.

$$
\begin{gathered}
A=\left[\begin{array}{cccc}
\frac{-R}{L} & -\omega & \frac{-6 \bar{V}_{\text {gird }}^{\alpha}}{2 L}+\frac{3 \bar{V}_{m}^{\alpha}}{2 L} & \frac{-6 \bar{V}_{\text {gird }}^{\beta}}{2 L}+\frac{3 \bar{V}_{m}^{\beta}}{2 L} \\
\omega & \frac{-R}{L} & -\frac{3 \bar{V}_{m}^{\beta}}{2 L} & \frac{3 \bar{V}_{m}^{\alpha}}{2 L} \\
0 & 0 & 0 & -\omega \\
0 & 0 & \omega & 0
\end{array}\right] \\
B=\frac{3}{2 L}\left[\begin{array}{cc}
\bar{V}_{\text {gird }}^{\alpha} & \bar{V}_{\text {gird }}^{\beta} \\
\bar{V}_{\text {gird }}^{\beta} & -\bar{V}_{\text {gird }}^{\alpha} \\
0 & 0 \\
0 & 0
\end{array}\right]
\end{gathered}
$$

where $\bar{z}$ denotes the currently available value of $z($.$) ,$ $z=V_{\text {gird }}^{\alpha}, V_{\text {gird }}^{\beta}, V_{m}{ }^{\alpha}, V_{m}{ }^{\beta}$.

$$
\begin{gathered}
D=\left[\begin{array}{cc}
-\frac{1}{R_{C 1} C_{1}} & 0 \\
0 & -\frac{1}{R_{C 2} C_{2}}
\end{array}\right], F=\left[\begin{array}{cc}
-\frac{1}{C_{1}} & 0 \\
0 & -\frac{1}{C_{2}}
\end{array}\right] \\
G=\left[\begin{array}{c}
\frac{1}{C_{1}} \\
\frac{1}{C_{2}}
\end{array}\right]
\end{gathered}
$$

\section{ACKNOWLEDGMENT}

The first author would like to acknowledge the financial support provided by the Australian Government Research Training Program Scholarship. 


\section{REFERENCES}

S. Vazquez, J. Rodriguez, M. Rivera, L. G. Franquelo, and M. Norambuena, "Model predictive control for power converters and drives: Advances and trends," IEEE Transactions on Industrial Electronics, vol. 64, pp. 935947, 2017

[2] J. I. Leon, S. Kouro, L. G. Franquelo, J. Rodriguez, and B. Wu, "The Essential Role and the Continuous Evolution of Modulation Techniques for Voltage-Source Inverters in the Past, Present, and Future Power Electronics," IEEE Transactions on Industrial Electronics, vol. 63, pp. 26882701, 2016.

[3] L. Wang, Model predictive control system design and implementation using MATLAB ${ }^{\circledR}$ : Springer Science \& Business Media, 2009.

[4] G. Papafotiou, G. Demetriades, and V. Agelidis, "Technology Readiness Assessment of Model Predictive Control in Medium-and High-Voltage Power Electronics," IEEE Transactions on Industrial Electronics, vol. 63, pp. 5805-5815, 2016.

[5] E. Babaei, C. Buccella, and M. Saeedifard, "Recent Advances in Multilevel Inverters and Their Applications-Part II," IEEE Transactions on Industrial Electronics, vol. 63, pp. 7777-7779, 2016.

[6] J. Scoltock, T. Geyer, and U. K. Madawala, "Model predictive direct power control for grid-connected NPC converters," IEEE Transactions on Industrial Electronics, vol. 62, pp. 5319-5328, 2015.

[7] R. P. Aguilera, P. Lezana, and D. E. Quevedo, "Switched model predictive control for improved transient and steady-state performance," IEEE Transactions on Industrial Informatics, vol. 11, pp. 968-977, 2015.

[8] T. J. Vyncke, S. Thielemans, and J. A. Melkebeek, "Finite-set model-based predictive control for flyingcapacitor converters: Cost function design and efficient FPGA implementation," IEEE Transactions on Industrial Informatics, vol. 9, pp. 1113-1121, 2013.

[9] F. Salinas, M. A. González, and M. F. Escalante, "Finite Control Set-Model Predictive Control of a Flying Capacitor Multilevel Chopper Using Petri Nets," IEEE Transactions on Industrial Electronics, vol. 63, pp. 5891$5899,2016$.

[10] R. Baidya, R. Aguilera, P. Acuna, S. Vazquez, and H. D. Mouton, "Multistep Model Predictive Control for Cascaded H-Bridge Inverters: Formulation and Analysis," IEEE Transactions on Power Electronics, 2017.

[11] S. S. Fazel, S. Bernet, D. Krug, and K. Jalili, "Design and comparison of 4-kV neutral-point-clamped, flyingcapacitor, and series-connected H-bridge multilevel converters," IEEE Transactions on Industry Applications, vol. 43, pp. 1032-1040, 2007.

[12] P. Cortés, S. Kouro, B. La Rocca, R. Vargas, J. Rodríguez, J. I. León, et al., "Guidelines for weighting factors design in model predictive control of power converters and drives," in Industrial Technology, 2009. ICIT 2009. IEEE International Conference on, 2009, pp. $1-7$.

[13] C. A. Rojas, J. Rodriguez, F. Villarroel, J. R. Espinoza, C. A. Silva, and M. Trincado, "Predictive torque and flux control without weighting factors," IEEE Transactions on Industrial Electronics, vol. 60, pp. 681-690, 2013.

[14] M. Siami, H. K. Savadkoohi, A. Abbaszadeh, D. Khaburi, J. Rodriguez, and M. Rivera, "Predictive torque control of a permanent magnet synchronous motor fed by a matrix converter without weighting factor," in Power Electronics and Drive Systems Technologies Conference (PEDSTC), 2016 7th, 2016, pp. 614-619.

[15] Y. Zhang and H. Yang, "Two-vector-based model predictive torque control without weighting factors for induction motor drives," IEEE Transactions on Power Electronics, vol. 31, pp. 1381-1390, 2016.

[16] F. Villarroel, J. R. Espinoza, C. Rojas, J. Rodriguez, M. Rivera, and D. Sbárbaro, "Multiobjective switching state selector for finite-states model predictive control based on fuzzy decision making in a matrix converter," Industrial Electronics, IEEE Transactions on, vol. 60, pp. 589-599, 2013.

[17] C. A. Rojas, S. Kouro, M. Perez, and F. Villarroel, "Multiobjective Fuzzy Predictive Torque Control of an induction machine fed by a 3L-NPC inverter," in 2015 IEEE International Symposium on Predictive Control of Electrical Drives and Power Electronics (PRECEDE), 2015, pp. 21-26.

[18] J. Scoltock, T. Geyer, and U. K. Madawala, "Model Predictive Direct Power Control for Grid-Connected NPC Converters," Industrial Electronics, IEEE Transactions on, vol. 62, pp. 5319-5328, 2015. 